\title{
Analyses des matériaux de construction bruts de Portus par la Spectroscopie Moyen InfraRouge (SMIR)
}

Application à l'étude du môle nord du port antique de Rome et création d'une base minéralogique spectrale

Stoil Chapkanski, Jean-Philippe Goiran, Carlo Rosa, Simon Keay, Stephen Kay, Damien Ertlen et Xavier Gallet

\section{(2) OpenEdition}

12 Journals

Édition électronique

URL : https://journals.openedition.org/baefe/3278

DOI : $10.4000 /$ baefe.3278

ISSN : 2732-687X

Éditeur

ResEFE

Référence électronique

Stoil Chapkanski, Jean-Philippe Goiran, Simon Keay, Carlo Rosa, Stephen Kay, Damien Ertlen et Xavier Gallet, «Analyses des matériaux de construction bruts de Portus par la Spectroscopie Moyen

InfraRouge (SMIR) » [notice archéologique], Bulletin archéologique des Écoles françaises à l'étranger [En ligne], Italie, mis en ligne le 21 juillet 2021, consulté le 23 juillet 2021. URL : http://

journals.openedition.org/baefe/3278; DOI : https://doi.org/10.4000/baefe.3278

Ce document a été généré automatiquement le 23 juillet 2021.

\section{cc) (†)}

Le Bulletin archéologique des Écoles françaises à l'étranger est mise à disposition selon les termes de la Licence Creative Commons Attribution - Pas d'Utilisation Commerciale - Pas de Modification 4.0 International. 


\section{Analyses des matériaux de construction bruts de Portus par la Spectroscopie Moyen InfraRouge (SMIR)}

Application à l'étude du môle nord du port antique de Rome et création d'une base minéralogique spectrale

Stoil Chapkanski, Jean-Philippe Goiran, Carlo Rosa, Simon Keay, Stephen Kay, Damien Ertlen et Xavier Gallet

\section{NOTE DE L'AUTEUR}

Dates précises des opérations : Carottage sédimentaire (mai 2017), prélèvements d'échantillons d'affleurements naturels (mai 2018) et mise en place de la base de données minéralogique spectrale (décembre 2018-mai 2019)

Autorité nationale présente : Parco Archeologico di Ostia Antica

Numéro de mission : N/A

Composition de l'équipe de terrain : Carottage sur le môle nord de Portus : JeanPhilippe Goiran, UMR 5133-Université de Lyon 2 ; Simon Keay, University of Southampton; Stephen Kay, British School at Rome ; Piero Bellotti, Sapienza Università di Roma ; Daniele D'Ottavio, Société de carottage Geoambiente. Prélèvements d'échantillons d'affleurements naturels à Rome : Stoil Chapkanski, UMR 5133, Université de Lyon 2 - CNRS et UMR 8591, Université de Paris 1, Panthéon Sorbonne ; Carlo Rosa, Instituto Italiano di Paleontologia UmanaIntroduction.

Partenariats institutionnels : Soprintendenza speciale per i Beni Archeologici di Roma, Parco Archeologico di Ostia Antica, la British School of Rome, l'Université de Southampton, et le Muséum National d'Histoire Naturelle MNHN, École Française de Rome, Université Aix-Marseille.

Établissement éditeur : EFR 
Établissements porteurs de l'opération : Université de Lyon 2, le Centre National de la Recherche Scientifique CNRS, l'Université de Southampton et École Française de Rome.

Remerciements : Ce travail est dédié à notre cher collègue, ami et co-auteur Simon Keay, professeur à l'Université de Southampton, qui a disparu avant la publication de cet article. Le travail a été partiellement financé par le projet du Conseil Européen de la Recherche (ERC) Portus-Limen, Rome's Mediterranean Ports, porté par Simon Keay, (numéro 339123), le projet IDEX - Thalassocraties sans ports: mythe ou réalité ?, le Soutien à la mobilité internationale SMI-INSHS et le programme Fosphora. Les auteurs souhaitent remercier la Soprintendenza speciale per i Beni Archeologici di Roma et le Parco Archeologico di Ostia Antica pour les autorisations de travail ainsi que Piero Bellotti (Sapienza Università di Roma) pour son expertise durant la mission de carottage et Gabriel Caroline pour son aide durant le tamisage des échantillons.

Données scientifiques produites :

https://portuslimen.eu/

https://amidex.hypotheses.org/9931

\section{Introduction}

La caractérisation de matériaux de construction et leur provenance sont généralement étudiées par des analyses pétrographiques, optiques, minéralogiques et géochimiques ${ }^{1}$. Efficace, rapide, non-destructive et dans certains cas plus précise que les techniques conventionnelles, la Spectroscopie Moyen Infrarouge (SMIR) à Transformée de Fourier (FTIR) est une approche alternative pour étudier les propriétés physiques, minéralogiques et biochimiques des roches, des sédiments et des matériaux de construction. Le principe de la spectroscopie implique la mesure de l'interaction entre la matière étudiée et le rayonnement infrarouge incident à différentes fréquences du spectre électromagnétique infrarouge ${ }^{2}$. La radiation infrarouge induit des vibrations moléculaires permettant une absorbance des molécules à certaines longueurs d'ondes spécifiques. L'ensemble des longueurs d'ondes constitue le spectre infrarouge qui reflète la composition moléculaire de la matière étudiée et par conséquent constitue l'empreinte digitale de l'échantillon.

2 Portus Claudius, le port impérial de Rome a été construit durant le $\mathrm{I}^{\mathrm{er}}$ siècle apr. J.-C. sur la rive droite du Tibre, environ $30 \mathrm{~km}$ au sud-ouest de Rome (fig. 1). Le bassin portuaire comprend deux grands môles qui enferment un espace portuaire d'environ 200 hectares. Aujourd'hui, les structures portuaires se retrouvent ensevelies à l'intérieur des terres suite au déplacement de la ligne de rivage et à l'apport des sédiments par la progradation du delta sur la $\mathrm{mer}^{3}$. Le flux sédimentaire terrigène a progressivement modifié les paysages et perturbe la compréhension de la configuration des structures portuaires. Néanmoins, des avancées scientifiques significatives dans la compréhension de l'extension nord du port ont été réalisées suite à des prospections lors de la construction de l'aéroport international Leonardo Da Vinci ${ }^{4}$. Ces avancées ont été complétées par des prospections géophysiques ${ }^{5}$ et des campagnes de carottages ${ }^{6}$. En outre, des investigations sur la composition du mortier utilisé pour la construction du 
môle nord de Portus ont été réalisées dans le cadre du projet ROMACONS en utilisant des carottes courtes (de 1,3 à $3 \mathrm{~m}^{7}$ ).

Fig. 1. Carte de localisation de Portus à différentes échelles spatiales.

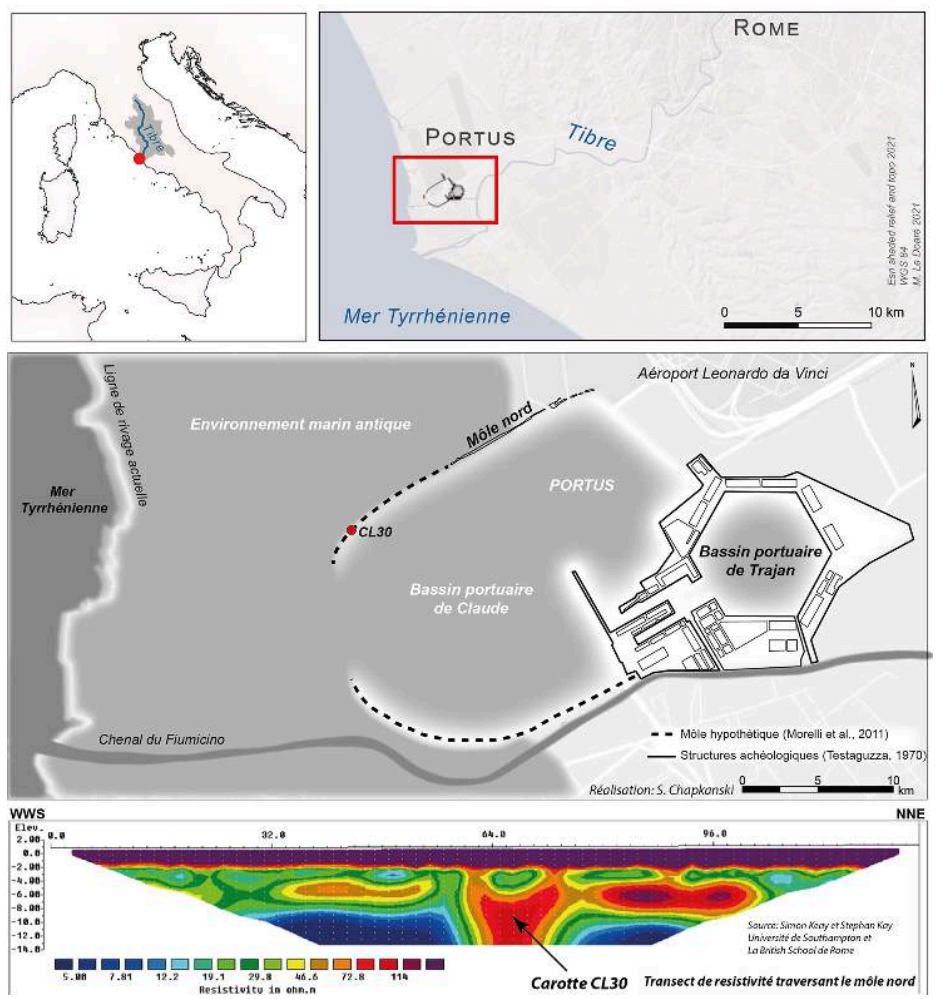

\section{Problématique et objectifs de la recherche}

Bien que la compréhension du Portus comme une infrastructure portuaire ait été grandement améliorée, la provenance lithologique des matériaux de construction bruts utilisés pour la construction des môles demeure inconnue. Par conséquent, en 2017 les structures ensevelies du môle nord de Portus ont été traversées par un carottage profond de $22 \mathrm{~m}$ lors d'une mission mise en place grâce à la coopération internationale entre les institutions (Conseil Européen de la Recherche(ERC) Portus-Limen, Soprintendenza speciale per i Beni Archeologici di Roma, la British School de Rome, l'Université de Southampton, le Centre national de la recherche scientifique CNRS et École Française de Rome dans le cadre du programme Ostie-Portus).

Depuis, une équipe pluridisciplinaire incluant des sédimentologues, géoarchéologues, archéologues et géologues a été mise en place afin de : (i) améliorer la compréhension de la composition et de la structure du môle nord et (ii) renseigner la provenance lithologique des matériaux de construction (dans le cadre du programme Fosphora). L'hypothèse de travail repose sur la possibilité de caractériser les matériaux de construction et d'identifier leur provenance grâce à leur signature spectrale. Pour tester l'hypothèse de travail, une approche impliquant des outils sédimentologiques, spectroscopiques et statistiques a été mise en place. 


\section{Matériels et méthodes}

\section{Carottage et échantillonnage}

5 Un carottage profond, nommé CL30, a été réalisé en utilisant un carottier mécanique rotatif dans les structures portuaires ensevelies, précédemment identifiées par des prospections géophysiques. La description stratigraphique détaillée incluant la couleur des sédiments, la texture, la dureté ainsi que la présence d'oxydation, de coquilles, de matière organique et de macro-restes, a été réalisée sur le terrain et synthétisée dans un $\log$ stratigraphique.

\section{Travail en laboratoire}

6 Les échantillons prélevés ont été séchés à l'étuve $\left(50^{\circ} \mathrm{C}\right)$ pendant 7 jours. Le pourcentage de texture (argiles et limons, sables, sédiments grossiers) a été réalisé à partir de $10 \mathrm{gr}$ de sédiments suite à un tamisage humide à $0,063 \mathrm{~mm}$ et $2 \mathrm{~mm}$. En outre, la susceptibilité magnétique a été réalisée en utilisant un appareil Bartington MS2. Les mesures spectroscopiques ont été réalisées sur des échantillons broyés en utilisant un spectromètre FT-IR Frontier (PerkinElmer). Afin de déterminer la technique spectroscopique infrarouge qui permet la meilleure identification des liaisons chimiques d'échantillons, la réflexion totale atténuée (ATR) et la réflexion diffuse à transformée de Fourier (DRIFT) ont été employées en comparaison (fig. 2). Après l'acquisition spectrale, la matrice de données a été standardisée et alignée à sa base en utilisant le logiciel Unscrambler (Camo Unscrambler 10.3). Des précisions techniques sont détaillées par S. Chapkanski et collaborateurs ${ }^{8}$. 
Fig. 2. Représentation schématique de l'acquisition du spectre IR selon deux techniques.

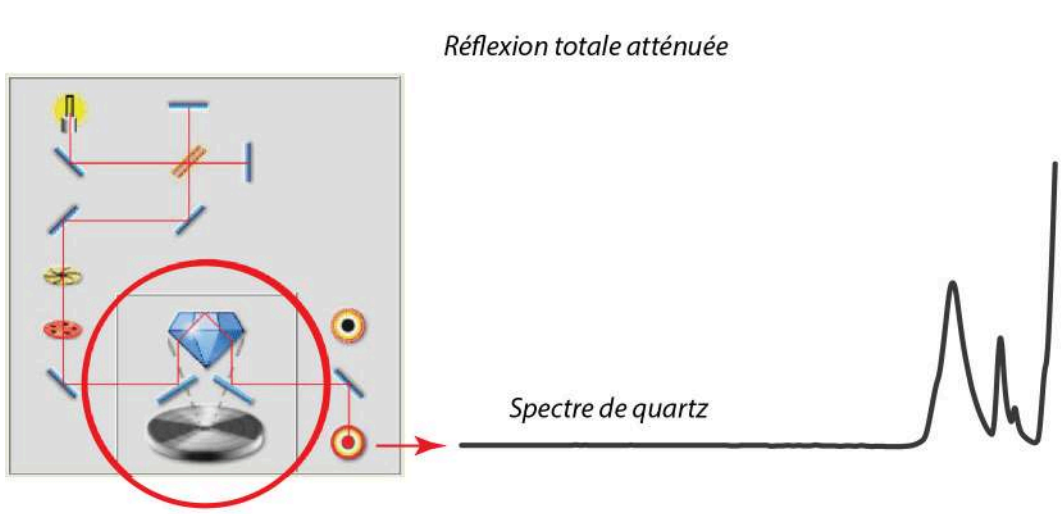

Réflexion diffuse à transformée de Fourier

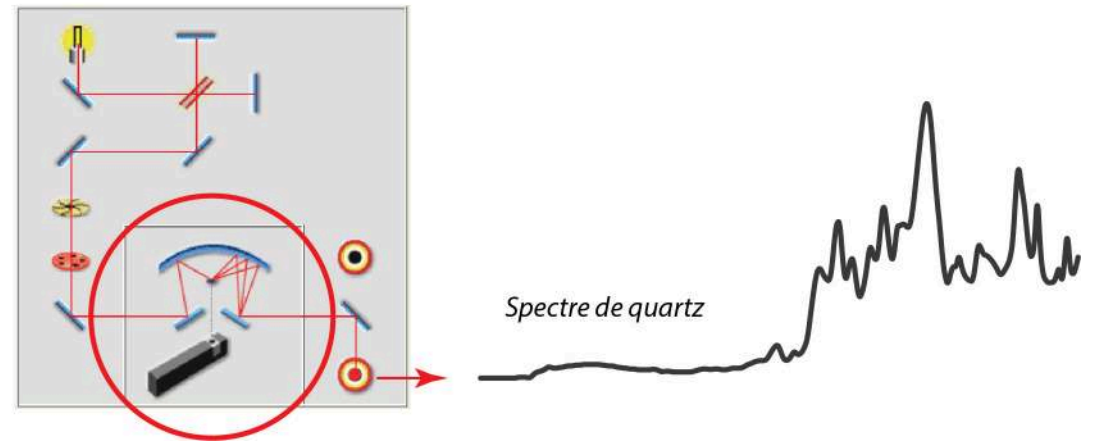

Le spectre de quartz est fourni comme un exemple.

Les images proviennent des manuels PerkinElmer.

\section{Élaboration d'une base minéralogique spectrale}

7 En parallèle de l'étude des matériaux de construction du môle nord, une base minéralogique spectrale incluant des minéraux purs et des roches de référence est en cours de développement en collaboration avec le Muséum national d'Histoire naturelle. Les spectres ont été enregistrés plusieurs fois dans des conditions ambiantes différentes avec deux techniques spectroscopiques (à savoir l'ATR et la DRIFT) afin de garantir la représentativité des spectres de référence (fig. 3). La base minéralogique spectrale devrait permettre une meilleure reconnaissance des pics d'absorbance et par conséquent une meilleure identification des composés minéralogiques présents dans l'échantillon. En outre, en suivant la méthode des ajouts dosés et la loi de LambertBeer', plus d'une centaine d'échantillons artificiels à teneur minéralogique dosée ont été créés afin de dresser une courbe d'étalonnage pour le quartz, la calcite, la kaolinite et le gypse. À terme, ces courbes permettront de quantifier la teneur de ces minéraux au sein d'un échantillon. 
Fig. 3. Aperçu de la base minéralogique spectrale.

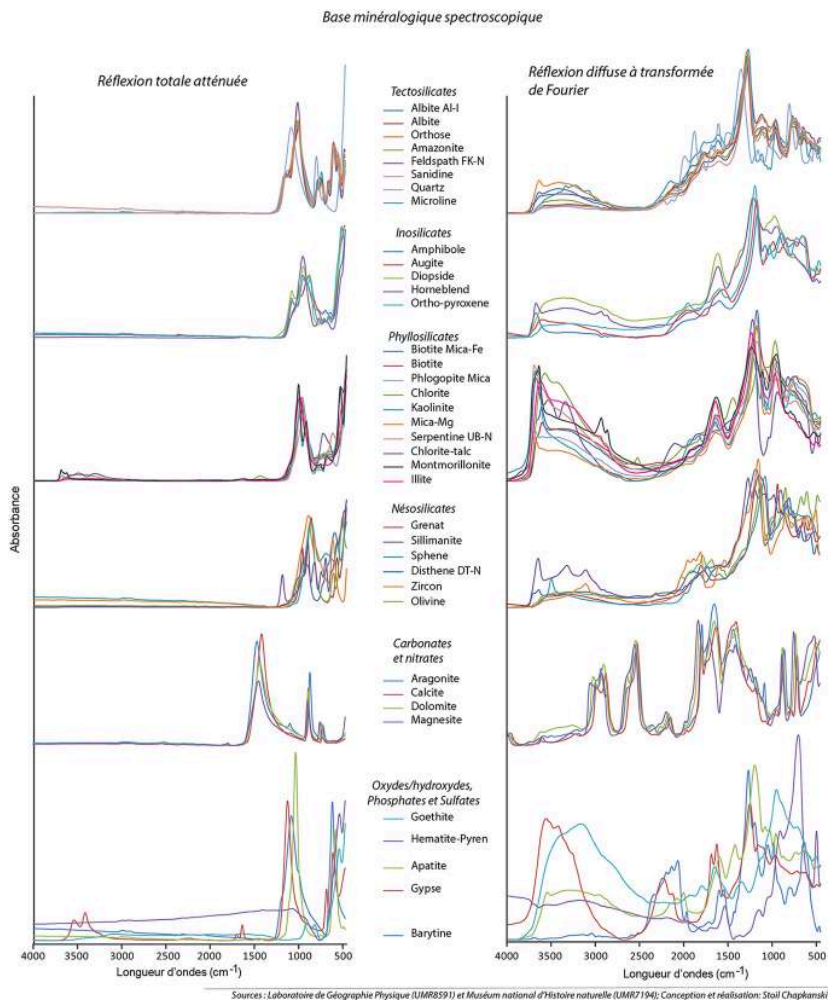

\section{Résultats préliminaires}

\section{Stratigraphie de la carotte CL30}

La stratigraphie de la carotte CL30 pourrait être synthétisée en 3 unités stratigraphiques principales, elles-mêmes subdivisées en 2-3 sous-unités (fig. 4). L'unité basale (-22 à $-18 \mathrm{~m}$ de profondeur) est composée d'argiles limoneuses grises représentant environ $80 \%$ du poids tamisé total. La susceptibilité magnétique (SM) indique des valeurs basses proches de 0 . L'unité stratigraphique $B$ se prolonge sur environ $10 \mathrm{~m}$ de profondeur et présente une structure très hétérogène, composée de fragments volcaniques grossiers et friables à la base (-18 à $-15 \mathrm{~m}$ de profondeur), d'agrégats pyroclastiques durs dans la partie médiane de l'unité $(-14,5$ à $-9,5 \mathrm{~m}$ de profondeur) et de fragments de roche magmatique surplombés par des tessons romains (-9,5 à $-7,5 \mathrm{~m}$ de profondeur). La SM indique une grande variabilité entre les échantillons avec des valeurs allant de 100 jusqu'à 2000 CGS (unité de mesure magnétique). L'unité $C$ présente des sables lités qui occupent environ $60-70 \%$ du poids total de l'échantillon. À sa base (sous-unité $\mathrm{C} 1$ ), les sables lités contiennent des fragments de coquilles brisées ( -7.5 à $-6 \mathrm{~m}$ de profondeur). Des sables grossiers gris légèrement oxydés occupent la partie médiane de l'unité (sous-unité $\mathrm{C} 2$ ). Cette dernière est surmontée par des sables limoneux lités de couleur jaune. La SM indique des valeurs relativement basses avec des pics magnétiques qui semblent fortement corrélés avec la granulométrie grossière, notamment les graviers fins. 
Fig. 4. Stratigraphie de la carotte CL30.

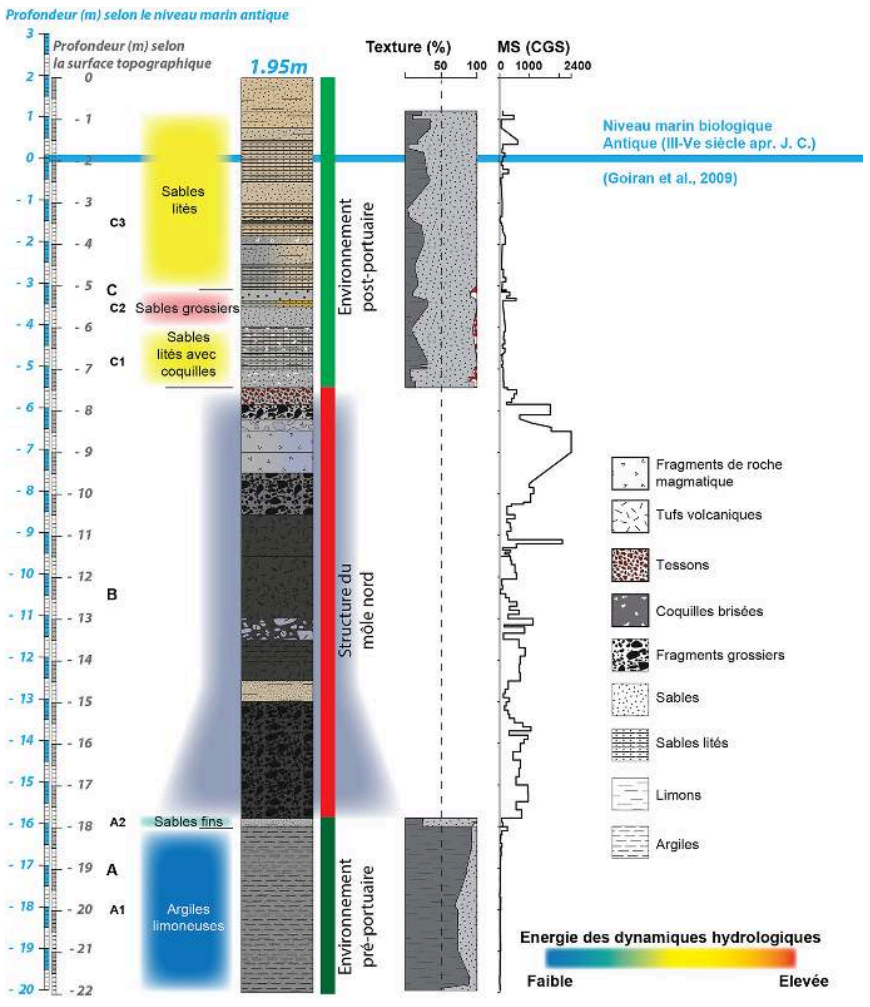

\section{Les matériaux bruts du môle nord}

9 Les observations des matériaux de construction utilisés pour la construction du môle nord de Portus révélés par la carotte CL30 ont montré la présence de tufs volcaniques Lionato (fig. 5, images P2, C2 et C4), de tufs Rosso a scorie nere sabatino (images P1 et C3), de pouzzolane (image C1) et de fragments de roches volcaniques ignées contentant des cristaux de leucite (images B1 et B2). En outre, des dépôts sableux peu consolidés ont également été retrouvés (image $\mathbf{S 1}$ ). 
Fig. 5. Aperçu des matériaux de construction prélevés à partir de la carotte CL30.

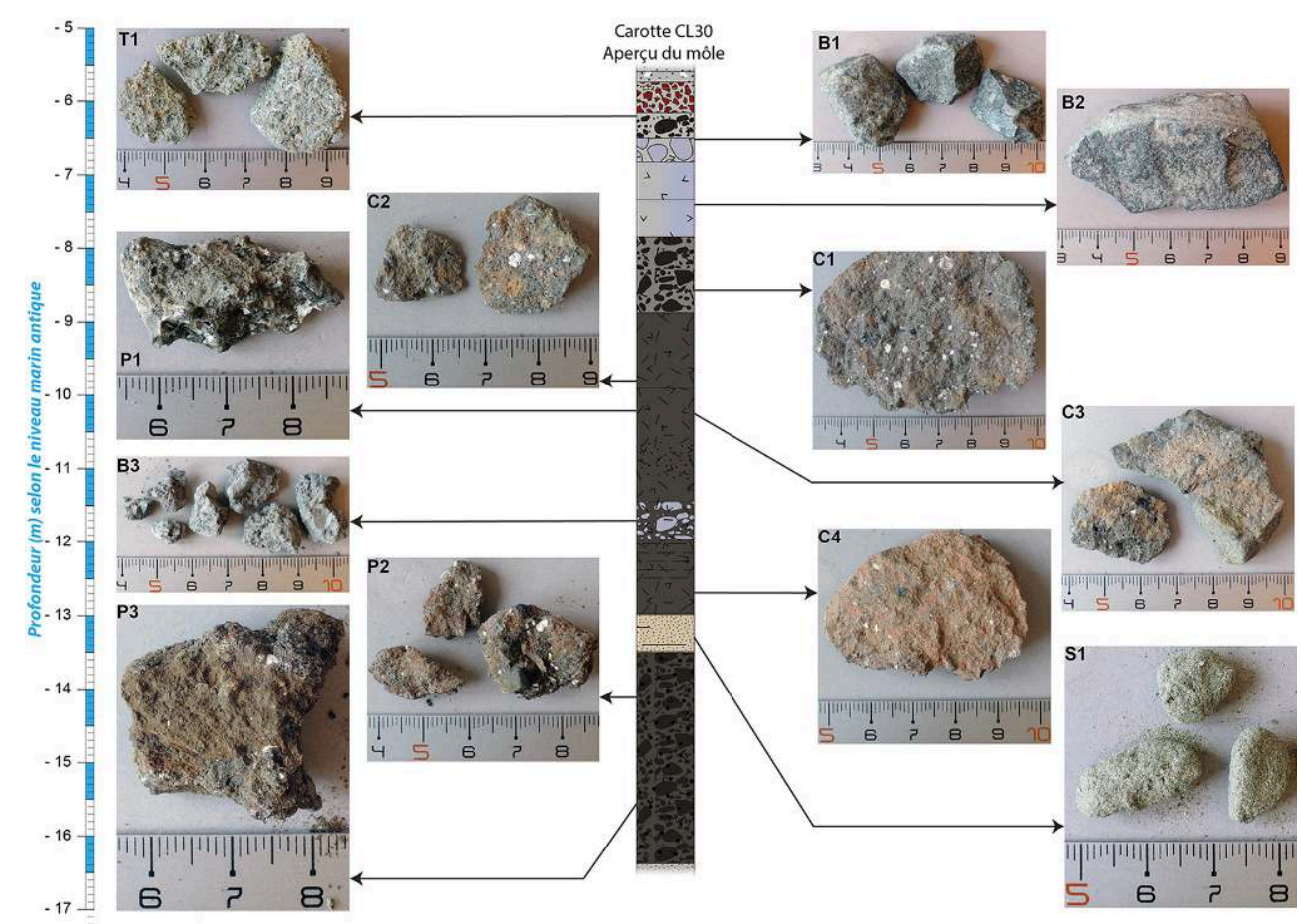

\section{La réponse spectrale des matériaux de construction}

Dans le cadre de cet article, seuls les résultats obtenus par la réflexion diffuse à transformée de Fourier seront présentés. La figure 6 donne un aperçu de quatre spectres obtenus à partir de l'analyse de différents matériaux utilisés pour la construction du môle. Les pics spectraux ont été identifiés grâce à la base minéralogique spectrale précédemment établie (fig. 3). Le spectre des dépôts sableux (image S1) montre une grande variabilité des pics et met en évidence de fortes teneurs en calcite et quartz (pics identifiés respectivement à $2500 \mathrm{~cm}-1$ et $1160 \mathrm{~cm}-1$ ). Les échantillons $\mathrm{C} 2$ et P2 ont été identifiés en tant que tufs volcaniques Lionato et leurs spectres mettent en évidence la présence de biotite ainsi que des tectosilicates notamment du feldspath dans la région de 1350 à $1200 \mathrm{~cm}-1$. L'échantillon B2, identifié comme fragment volcanique igné contentant des cristaux de leucite, met en évidence une réponse spectrale similaire aux tufs. Néanmoins, des variations spectrales sont identifiables dans la région spectrale dite " Fingerprint » située entre 1200 et $450 \mathrm{~cm}-1$. Afin de mieux comprendre les variations spectrales entre les échantillons qui sont difficilement perceptibles par observation visuelle, les spectres ont été examinés par l'analyse en composantes principales (ACP) afin de mettre en évidence le groupement naturel des matériaux investigués en fonction de leur composition minéralogique (fig. 7). L'ACP a produit sept axes dont les deux premiers résument $84 \%$ de la variance totale. La première composante met en évidence une opposition entre des tufs (à droite) et des fragments volcaniques ignés contenant des cristaux de leucite (à gauche). La deuxième composante met en évidence l'opposition entre les tufs à la base de la carotte (en haut sur le nuage de points) et les tufs au milieu de la structure portuaire. Cette opposition est en partie due à une plus forte concentration en calcite des tufs au milieu de la carotte (fig. 8). 
Fig. 6. Aperçu de la réponse spectrale de différents matériaux.

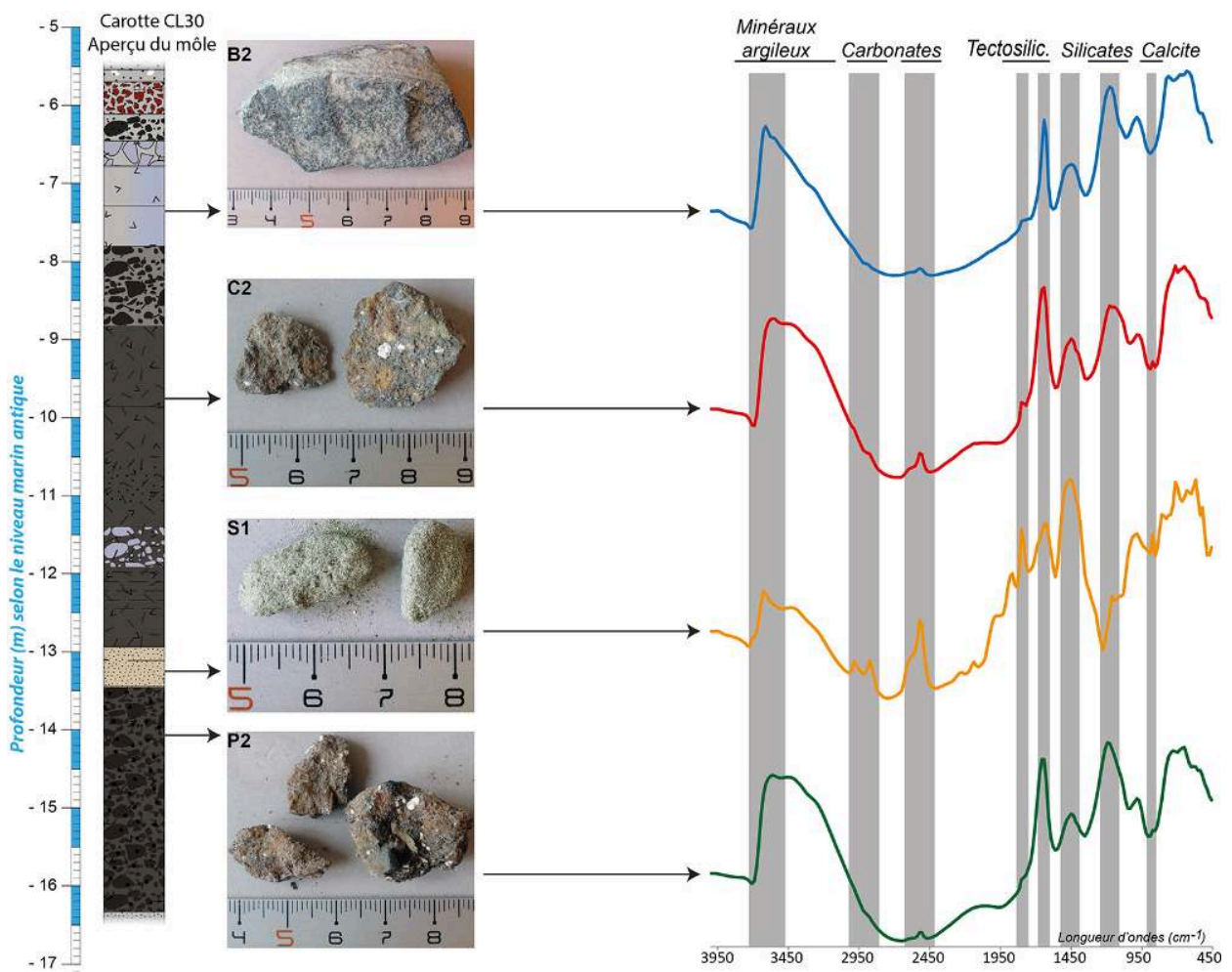

Des pics significatifs ont été soulignés.

Fig. 7. Nuage de points des réponses spectrales des matériaux de construction obtenu à partir des scores de l'Analyse en Composantes Principales.

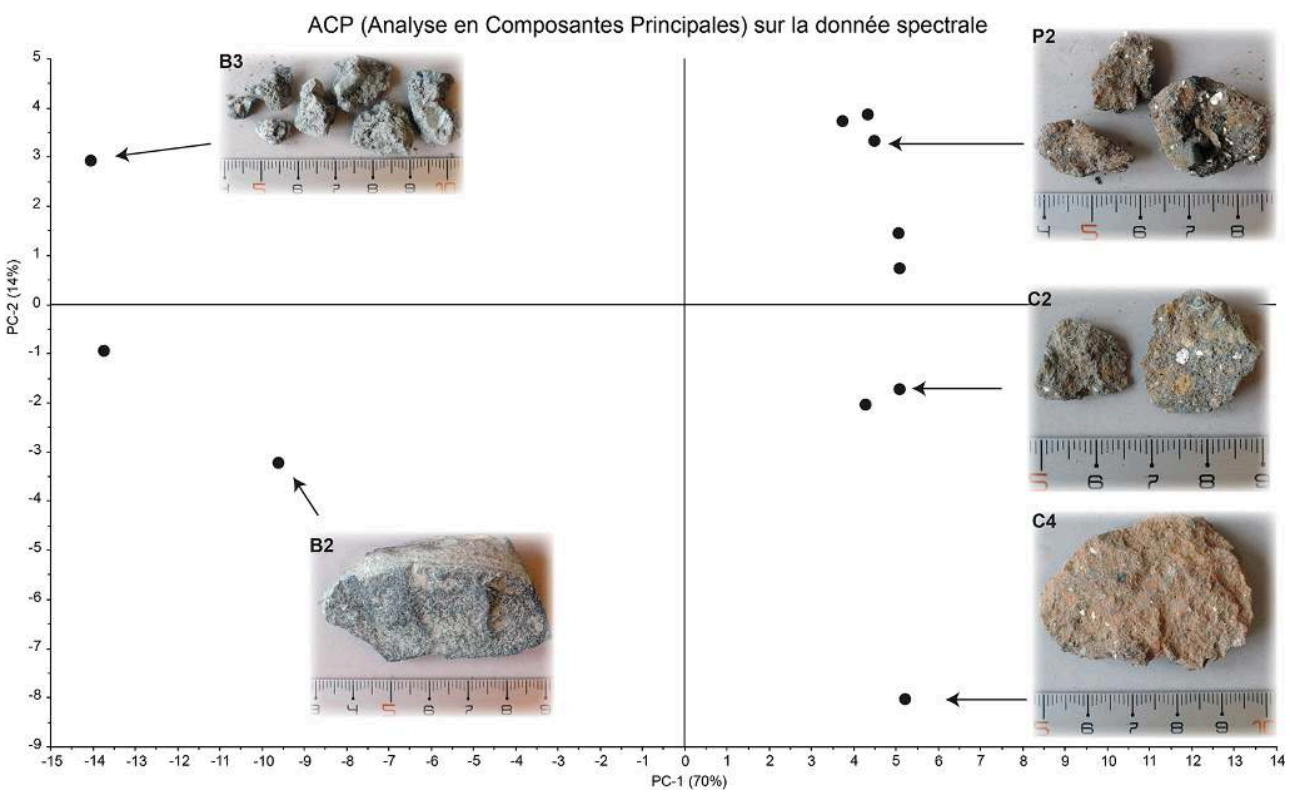


Fig. 8. Agrandissement sur une bande d'absorbance de la calcite.

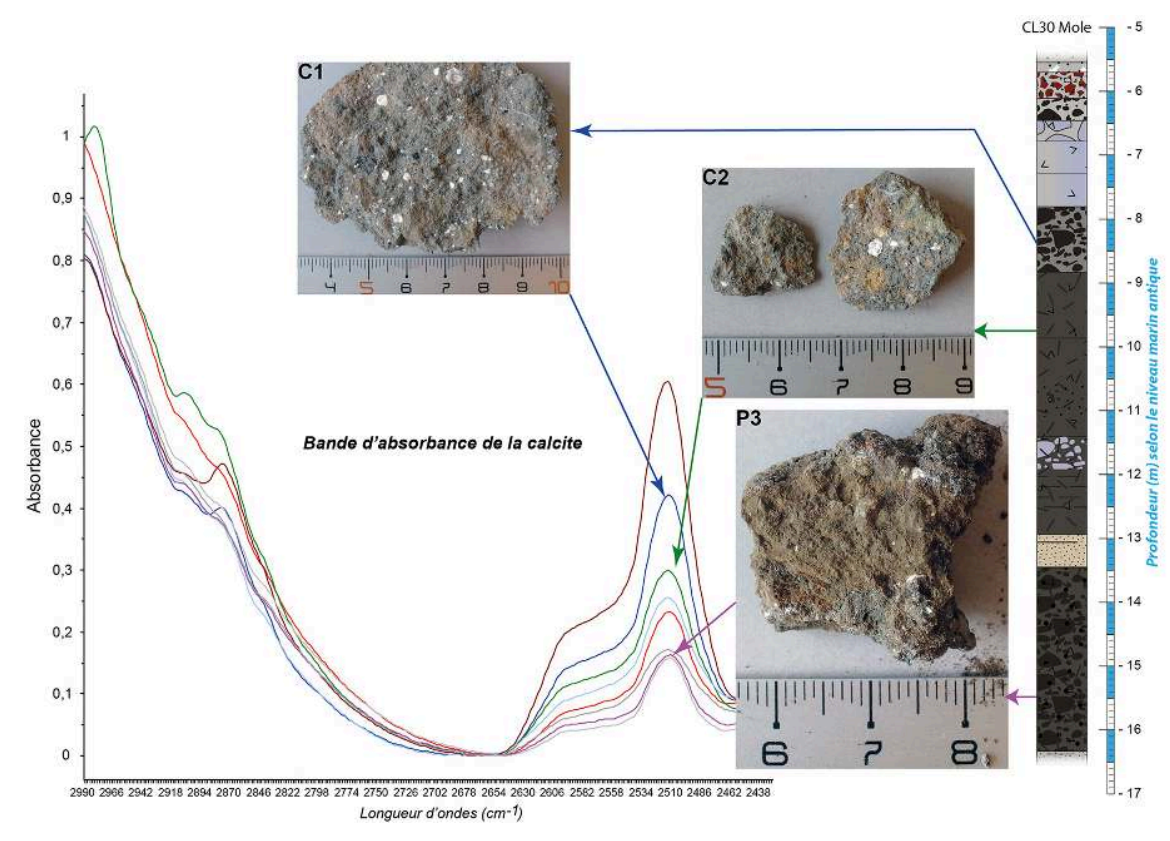

\section{Conclusion et perspectives}

11 Cet article permet de présenter les résultats préliminaires issus de l'investigation spectroscopique des matériaux de construction du môle nord de Portus. Bien que le nombre d'échantillons se limite à une quinzaine, ceux-ci montrent clairement le potentiel de la spectroscopie infrarouge pour distinguer les différents types de matériaux bruts et les regrouper en fonction des similarités minéralogiques. Ces résultats ont conforté les hypothèses émises et ont constitué un point de départ pour la suite des investigations. Par conséquent, l'équipe de travail a triplé le nombre d'échantillons de la carotte afin de proposer une analyse spectroscopique fine de la séquence du môle. De plus, un référentiel incluant des affleurements de tufs et de pouzzolane aux alentours de Rome (fig. 9) a été constitué afin de comparer les matériaux retrouvés au sein de la structure portuaire et les possibles sources lithologiques naturelles. Les résultats sont en cours de traitement avec l'objectif d'être publiés durant la deuxième moitié de 2021. En complément de cette étude, la base minéralogique spectrale permettrait une meilleure compréhension des matériaux de construction, notamment leurs propriétés minéralogiques et biochimiques. En outre, les résultats prometteurs issus des courbes d'étalonnage pour le quartz, la calcite, la kaolinite et le gypse permettraient de compter sur un protocole de quantification afin de détailler la teneur minéralogique des matériaux de construction.

L'approche présentée dans ce manuscrit pourra être transposée sur d'autres carottes de môle à Portus dans le cadre du programme Fosphora, afin de contribuer à une réflexion globale de la structure portuaire antique. 
Fig. 9. Localisation des prélèvements d'affleurements dans les environs de Rome.

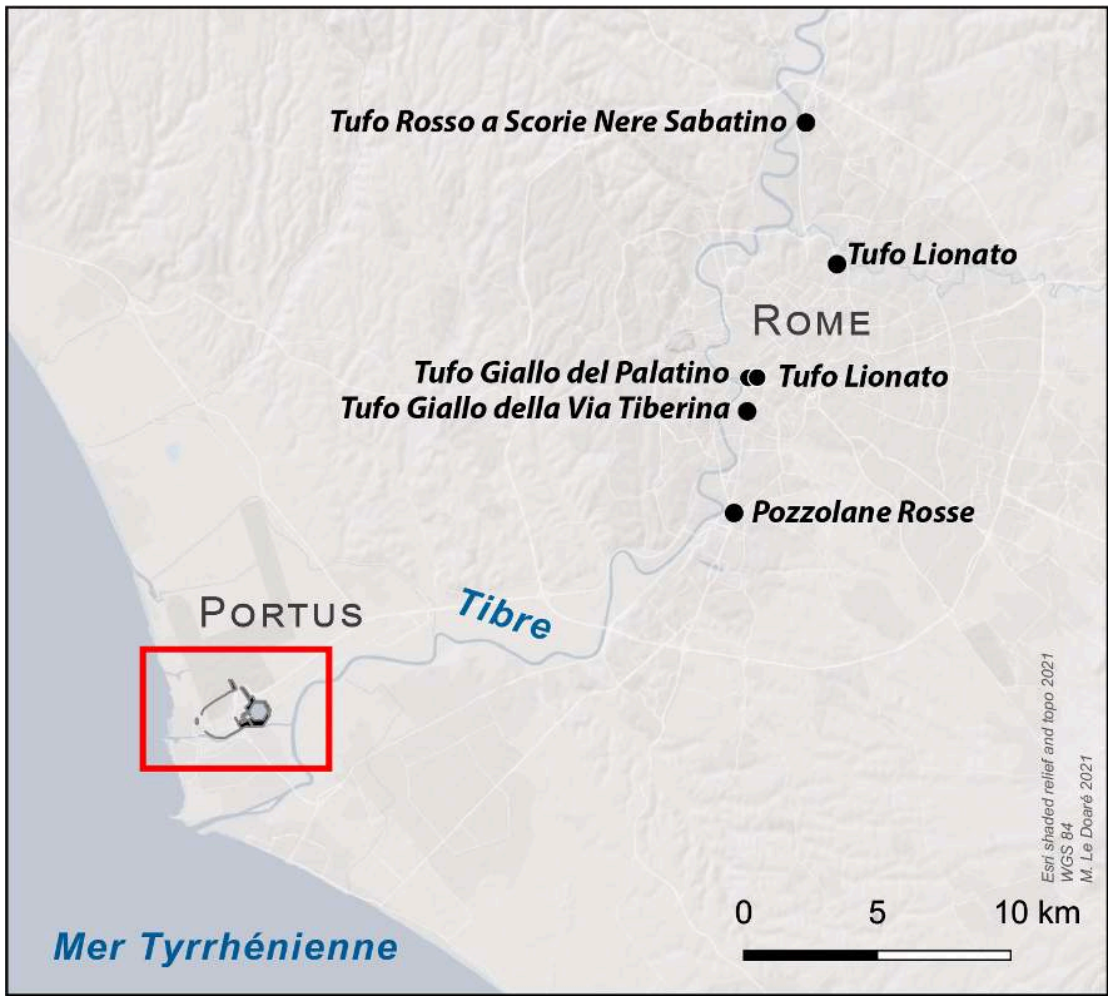

\section{BIBLIOGRAPHIE}

CHAPKANSKI et al. 2020

S. Chapkanski, D. Ertlen, C. Rambeau, L. Schmitt, « Provenance discrimination of fine sediments by mid-infrared spectroscopy: Calibration and application to fluvial palaeo-environmental reconstruction ", Sedimentology 67.2, 2020, p. 1114-1134.

FRÖHLICH, GENDRON-BADOU 2002

F. Fröhlich, A. Gendron-Badou, « La spectroscopie infrarouge, un outil polyvalent », Géologie de la Préhistoire, 2002, p. 662-677.

GIRAUDI 2011

C. Giraudi, « La geologia dell'Agro Portuense nell'ambito dell'evoluzione del delta del Tevere », in Keay, Paroli 2011, p. 21-30.

KEAY, PAROLI 2018

Simon Keay, Lidia Paroli (dir.), Portus and its Hinterland: Recent Archaeological Research, Londres, British School at Rome, 2011.

KEAY, KAY 2018

S. Keay et S. Kay, " The Roman Ports Project ", Papers of the British School at Rome 86, 2018,

p. 317-320. 
MORELLI, MARINUCCI, ARNOLDUS-HUYZENDVELD 2011

C. Morelli, A. Marinucci, A. Arnoldus-Huyzendveld, « Il Porto di Claudio: nuove scoperte », in Keay, Paroli, 2011, p. 47-65.

OLESON et al. 2004

J.P. Oleson, C. Brandon, S. Cramer, R. Cucitore, E. Gotti, R. Hohlfelder, « The ROMACONS Project: A contribution to the historical and engineering analysis of hydraulic concrete in Roman maritime structures », International Journal of Nautical Archaeology 33.2, 2004, p. 199-229.

PEZZOLo et al. 2018

A.D.L. Pezzolo, M. Colombi, G.A. Mazzocchin, « Where did Roman masons get their material from? A preliminary DRIFTS/PCA investigation on mortar aggregates from X Regio buildings in the Veneto area (NE Italy) and their potential sources ", Environmental Science and Pollution Research 25.29, 2018, p. 28798-28807.

PICHARD, FROHLICH 1986

C. Pichard, F. Frohlich, « Analyses IR quantitatives des sédiments. Exemple du dosage du quartz et de la calcite », Revue de l'Institut français du pétrole 41.6, 1986, p. 809-819.

TESTAGUZZA 1970

O. Testaguzza, « The port of Rome », Archaeology 17.3, 1970, p. 173-179.

\section{NOTES}

1. PEZZOLO et al. 2018, p. 28798-28799.

2. FRÖHLICH, GENDRON-BADOU 2002, p. 662-663.

3. GIRAUDI 2011, p. 21.

4. TESTAGUZZA 1970.

5. KEAY, KAY 2018, p. 317-320.

6. MORELLI et al. 2011, p. 56-58 ; GOIRAN et al. 2010, p. 5-11.

7. OLESON et al. 2004, p. 210-213.

8. CHAPKANSKI et al. 2020, p. 1119-1121.

9. PICHARD, FROHLICH 1986, p. 811-813. 
INDEX

Année de l'opération : 2018

Thèmes : EFR

chronologie https://ark.frantiq.fr/ark:/26678/pcrtZTmusVUU24

sujets https://ark.frantiq.fr/ark:/26678/pcrtI7E5QGYFhb, https://ark.frantiq.fr/ark:/26678/ pcrtVemT606YVG, https://ark.frantiq.fr/ark:/26678/pcrtwh5icnwutJ, https://ark.frantiq.fr/ ark:/26678/pcrtrMs1qgrvxu

lieux https://ark.frantiq.fr/ark:/26678/pcrtW9ctTWwSAK

\section{AUTEURS}

\section{STOIL CHAPKANSKI}

Archéorient, UMR 5133, Université de Lyon 2 - CNRS, 7 rue Raulin, 69007 Lyon, France ; Laboratoire de Géographie Physique (LGP), UMR 8591, Université de Paris 1, Panthéon Sorbonne - CNRS, 1 place Aristide Briand, 92195 Paris, France, (stoil.chapkanski@lgp.cnrs.fr / stoil.chapkanski@protonmail.com)

\section{JEAN-PHILIPPE GOIRAN}

Archéorient, UMR 5133, Université de Lyon 2 - CNRS, 7 rue Raulin, 69007 Lyon, France

\section{CARLO ROSA}

Instituto Italiano di Paleontologia Umana, Via Ulisse Aldrovandi, 18, 00197 Roma RM, Italy

\section{SIMON KEAY}

University of Southampton, Department of Archaeology, Faculty of Humanities, Avenue Campus, SouthamptonSO17 1BF, UK

\section{STEPHEN KAY}

British School at Rome, Via Antonio Gramsci, 61, 00197 Roma RM, Italy

\section{DAMIEN ERTLEN}

Laboratoire Image Ville Environnement (LIVE), UMR 7362, Université de Strasbourg - CNRS ENGEES, 3 Rue de l'Argonne, 67083 Strasbourg, France

\section{XAVIER GALLET}

Unité Mixte de Recherche 7194, Histoire Naturelle de l'Homme Préhistorique (HNHP), CNRS, Muséum National d'Histoire Naturelle, Université Perpignan Via Domitia - Sorbonne Universités, 17 Place du Trocadéro, F-75116 Paris, France 